\title{
Odor selectivity of hyposmia and cognitive impairment in patients with Parkinson's disease
}

\author{
This article was published in the following Dove Press journal: \\ Clinical Interventions in Aging \\ 9 October 2017 \\ Number of times this article has been viewed
}

\author{
Cheng-Jie Mao' \\ Fen Wang ${ }^{2}$ \\ Ju-Ping Chen ${ }^{3}$ \\ Ya-Ping Yang' \\ jing Chen' \\ Juan-Ying Huang' \\ Chun-Feng Liu' ${ }^{1,2}$ \\ 'Department of Neurology and \\ Suzhou Clinical Research Center of \\ Neurological Disease, the Second \\ Affiliated Hospital of Soochow \\ University, ${ }^{2}$ Institute of Neuroscience, \\ Soochow University, Suzhou, \\ ${ }^{3}$ Department of Neurology, Hospital \\ of Changshu Traditional Chinese \\ Medicine, Changshu, People's Republic \\ of China
}

Objective: Hyposmia is one of the earliest non-motor features of Parkinson's disease (PD) and can precede the onset of motor symptoms by years. Most of the current olfactory detection tests are targeted at Western populations. The exact relationship between hyposmia and cognitive impairment is unknown. The purpose of the study was to find bromines that can effectively identify olfactory dysfunction and investigate the relationship between hyposmia and cognitive function in early, non-demented, drug-naïve patients with PD in the People's Republic of China.

Methods: Sixty-three early, non-demented, drug-naïve patients with PD and 55 healthy controls were enrolled in the study. The T\&T olfactometer and a Chinese version of Montreal Cognitive Assessment (MoCA) were applied to assess subjects' olfactory and cognitive functions. Patients with PD also completed the Modified Unified Parkinson's disease-rating scale (UPDRS) and Hoehn and Yahr (H\&Y) scale.

Results: Patients with PD had lower scores of visuospatial and executive function $(p=0.000)$, attention ( $p=0.03$ ), and delayed recall $(p=0.001)$ than controls. $\beta$-phenylethyl alcohol (floral smell, smell of rose petals) and isovaleric acid (smell of sweat, stuffy socks) were more sensitive for identifying hyposmia in patients with PD than three other odors. Multivariate logistic regression analysis showed that impaired visuospatial and executive function was associated with hyposmia ( $p=0.013$ ), but was independent of other PD-associated variables.

Conclusion: Hyposmia was common in early, non-demented, drug-naïve PD patients. $\beta$-Phenylethyl alcohol and isovaleric acid were more superior for identifying hyposmia in early non-demented Chinese patients with PD. Hyposmia was associated with impaired visuospatial and executive function in patients with PD. Further prospective studies that apply a series of neuropsychological tests and functional magnetic resonance imaging methods in large samples in multicenter studies are needed to confirm our findings and to investigate the relationship between hyposmia and cognitive function with disease progression in patients with PD.

Keywords: Parkinson's disease, hyposmia, cognitive dysfunction

\section{Introduction}

Hyposmia is a term that describes a reduced ability to smell and to detect odors. Hyposmia is one of the earliest non-motor features of Parkinson's disease (PD). It can precede the onset of motor symptoms by years. ${ }^{1}$ Previous studies reported that approximately $80 \%-90 \%$ of patients with PD suffer from hyposmia. ${ }^{2,3}$ Hyposmia can be used as a biomarker for differentiating Alzheimer's disease (AD) and PD from progressive nuclear paralysis (PSP) and cortical basal ganglia degeneration (CBD) among neurodegenerative diseases. ${ }^{4,5}$ Hyposmia was reported to be correlated with cognitive impairment in some diseases, such as AD, PD, multiple sclerosis, and type 2 diabetes mellitus. ${ }^{6-9}$ Some studies revealed that hippocampal 
dopaminergic denervation, more than amygdala or striatal dopaminergic denervation, was correlated with impaired odor identification in early PD. ${ }^{10}$ Limbic cholinergic denervation may also contribute to hyposmia in PD. ${ }^{11}$ Progressive hyposmia may be used as a biomarker of cognitive decline and cholinergic denervation in patients with PD. ${ }^{12}$ But, the exact relationship between hyposmia and cognitive impairment in PD has not been elucidated. Patients with hyposmia were reported to have lower quality of life and higher mortality rates. ${ }^{13,14}$

There are several methods of olfactory testing, including the University of Pennsylvania Smell Identification Test (UPSIT), the Barcelona Smell test-24 (BAST-24) for Spain, "Screening 12 Test", and "Sniffin" Sticks'16 (SS-16) for olfactory testing. But not all odor-identification tests are applicable in different countries because of cultural differences. Most of the current olfactory tests are targeted toward Western populations. Only the Odor Stick Identification Test for Japanese (OSIT-J) was developed for Japanese populations. $^{15}$

There are two aims of this study. First, to identify bromines that can effectively identify olfactory dysfunction in Eastern populations, especially in patients with early PD in the People's Republic of China. Second, to explore the relationship between hyposmia and cognitive function in early non-demented patients with PD. Both of these aims are critical to improving early intervention and treatment strategies for patients with PD.

\section{Materials and methods Subjects}

A total of 63 eligible patients were selected from a cohort of consecutive Chinese patients diagnosed with PD in our movement disorder center from May 2014 to October 2015 whose Hoehn and Yahr (H\&Y) stage was 2.0 or lower. ${ }^{16}$ All of the PD subjects satisfied the UK Parkinson's Disease Society Brain Bank clinical diagnostic criteria. ${ }^{17}$ The Mini-Mental State Examination (MMSE) was applied as a screening tool for dementia in patients with PD and in controls. Subjects 45-80 years old with MMSE score $\geq 24$ (or $\geq 20$ if the patient had $<6$ years of education) who speak fluent Chinese and have no difficulty in communicating were enrolled in the study. ${ }^{18}$ Subjects were excluded if they had a secondary parkinsonism syndrome, atypical parkinsonian syndrome, malignant neoplasm, epilepsy, severe cardiopulmonary disease, a history of nasal or sinus or surgery, obstructive pulmonary disease, or had a cold in the 3 weeks preceding this study enrolment. Patients with MMSE score $<24$ (or $<20$ if the patient had $<6$ years of education) were excluded because odor-identification deficits are due, in large or small part, to impairment in verbal-semantic processing and how we learn about odors and their names. Prominent cognitive impairment could increase a perceptual basis of odor-identification failure. ${ }^{5,18-20}$ Fifty-five sex- and age-matched subjects were enrolled as controls.

\section{Procedures}

\section{Demographics and motor function evaluation}

Demographic information, including age, sex, years of education, disease duration, concurrent diseases, cigarette smoking, and detailed medical history, was collected. All subjects were carefully evaluated by a movement disorder specialist. The Modified Unified Parkinson's Disease Rating Scale (UPDRS) and Hoehn and Yahr (H\&Y) scale were applied to all subjects with PD. ${ }^{21}$

\section{Cognitive assessment}

A Chinese version of the Montreal Cognitive Assessment (MoCA; Beijing version), based on the final English language version was used in this study (Beijing version and English version of MoCA are available at http://www. mocatest.org with no permission requirements as declared). MoCA is a 30-point test covering seven cognitive subdomains: 1) visuospatial and executive; 2) naming; 3) memory; 4) attention; 5) language; 6) abstraction; and 7) delayed recall and orientation. A bonus point is given to individuals with $<12$ years of education. MoCA $<26$ is considered to be indicative of MCI; otherwise, it indicates normal cognition function, which was reported with a sensitivity of $90 \%$ and specificity of $87 \% .^{22}$

\section{Olfactory testing}

The T\&T Olfactometer (First Pharmaceutical Co., Ltd.) test, developed in Japan, has odor items that are easily recognizable to many Eastern people. ${ }^{23}$ The feature of this olfactory test with the T\&T Olfactometer is that the test simulates the action of sniffing in everyday among Asians. Therefore, this olfactory test should be useful in testing olfactory function in subjects with MMSE score $\geq 24$ (or $\geq 20$ if the patient has $<6$ years of education) who speak fluent Chinese and have no difficulty in communicating. The T\&T Olfactometer consists of five different types of odors, labeled A, B, C, $\mathrm{D}$, and $\mathrm{E}$, that have different chemical compositions of $\beta$-phenylethyl alcohol (floral smell, smell of rose petals), methyl cyclopentenolone (sweet burning smell, pudding), isovaleric acid (smell of sweat, stuffy socks), $\gamma$-undecalactone 
(smell of ripe fruit, canned peaches), 3-methylindole (musty smell, smell of feces). Each odor has five different concentrations. The sequential dilutions of each odor are as follows: $\mathrm{A}\left(10^{-3.0}, 10^{-3.5}, 10^{-4.0}, 10^{-4.5}\right.$, and $\left.10^{-5.0}\right)$; $\mathrm{B}\left(10^{-3.5}, 10^{-4.0}, 10^{-4.5}\right.$, $10^{-5.0}$, and $\left.10^{-5.5}\right)$; $\mathrm{C}\left(10^{-4.0}, 10^{-4.5}, 10^{-5.0}, 10^{-5.5}\right.$, and $\left.10^{-6.0}\right)$; $\mathrm{D}\left(10^{-3.5}, 10^{-4.0}, 10^{-4.5}, 10^{-5.0}\right.$, and $\left.10^{-5.5}\right)$, and $\mathrm{E}\left(10^{-4.0}, 10^{-4.5}\right.$, $10^{-5.0}, 10^{-5.5}$, and $\left.10^{-6.0}\right)$, respectively. The standard concentration for identification threshold of each odor are as follows: A $\left(3.92 \times 10^{-4.0}\right)$; B $\left(4.38 \times 10^{-4.5}\right)$; C $\left(4.92 \times 10^{-5.0}\right)$; $\mathrm{D}\left(4.35 \times 10^{-4.5}\right)$, and $\mathrm{E}\left(4.97 \times 10^{-5.0}\right)$, respectively. The concentration " 3 " represents the standard odor solutions. The weakest level is 1 , and the strongest is 5 . If the subject cannot detect or recognize the odor at the highest concentration, the result is labeled as 6. For each of the five different odors, the subject must state when the odor is first detected. That concentration is taken as the detection threshold value for that particular odor. The subject then continues to smell the five odors at increasing concentrations until the types of smell can actually be recognized. This is the subjects' identification threshold value for that particular odor. Subjects who are considered to have normal olfaction in the sensory test of standard odors have olfactory acuity values that are at least 1.5 standard deviations (SDs) under the mean. [https:// www.env.go.jp/en/air/odor/olfactory $\mathrm{mm} / 01 \mathrm{method} 2-1$. pdf]. The odor-identification threshold is the main observational indicator in this study. Subjects who recognized any of the five odors with a concentration higher than " 3 " were considered to have hyposmia.

The test was carried out in all patients with PD and controls in an odorless room. The examiner dips a test paper into the standard odor liquid to a depth of approximately $1 \mathrm{~cm}$ and passes it to the subject. To smell the strip, the subject should hold the tip of the paper approximately $1 \mathrm{~cm}$ away from the nose.

This study was approved by the ethics committee of the Second Affiliated Hospital of Soochow University. Each subject provided written informed consent before study participation.

\section{Statistics}

Continuous variables were expressed as mean \pm SD or median [interquartile range (IQR)], and were compared using Student's $t$-test or Wilcoxon rank-sum test between patients with PD and controls. Categorical variables were expressed as frequency (\%) and were compared using the chi-square or Fisher exact test. Multivariate non-conditional logistic regression models were used to assess the association of hyposmia with cognitive function and other variables in patients with PD. Odds ratios (ORs) and 95\% confidence intervals (95\% CIs) were used to evaluate the risk of hyposmia after adjusting for important confounders such as age and cigarette smoking. In addition, a receiver operating characteristic (ROC) analysis was conducted to assess the sensitivity and specificity of each odor for differentiating hyposmia. The area under the ROC curve (AUC) served as one primary index of accuracy. All $p$-values were twotailed, and a significance level of 0.05 was used. Statistical analysis was conducted using SPSS version 21 (IBM SPSS, Chicago, IL, USA).

\section{Results}

Sixty-three patients were included in this study. The baseline characteristics are presented in Table 1. The control group comprised 55 age- and sex-matched subjects. The mean age of PD patients was $62.7 \pm 7.4$ years. The median disease duration and H-Y stage were 2.0 years (IQR 1.0-7.0 years) and 1.5 (IQR 1.0-2.0), respectively. The mean score of

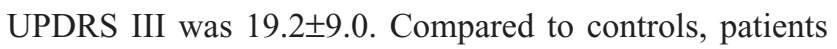
with PD had lower scores of MMSE $(p<0.001)$ and MoCA $(p<0.001)$. In terms of cognitive subdomains, patients with PD have lower scores of visuospatial and executive function $(p<0.001)$ and attention $(p=0.03)$, as well as delayed recall $(p=0.001)$, than controls.

Table I Baseline characteristics of patients with Parkinson's disease and controls

\begin{tabular}{llll}
\hline Characteristic & $\begin{array}{l}\text { PD patients } \\
(\mathbf{n}=63)\end{array}$ & $\begin{array}{l}\text { Controls } \\
(\mathbf{n}=\mathbf{5 5})\end{array}$ & p-value \\
\hline Age, years & $62.7 \pm 7.4$ & $62.9 \pm 7.0$ & 0.894 \\
Male sex & 40 & 34 & $0.85 \mathrm{I}$ \\
Education, years & $9.0(8.0-12.0)$ & $12.0(9.0-12.0)$ & $0.12 \mathrm{I}$ \\
Cigarette smoking & 22 & 16 & 0.499 \\
Disease duration, years & $2.0(1.0-7.0)$ & - & \\
Hoehn-Yahr stage & $1.5(1.0-2.0)$ & - & \\
UPDRS III & $19.2 \pm 9.0$ & - & \\
MMSE & $28.0(27.0-29.0)$ & $29.0(29.0-30.0)$ & $<0.001$ \\
MoCA & $24.0(22.0-26.0)$ & $26.0(24-27.0)$ & $<0.00 \mathrm{I}$ \\
Visuospatial and executive & $3.0(3.0-5.0)$ & $5.0(4.0-5.0)$ & $<0.00 \mathrm{I}$ \\
Naming & $3.0(3.0-3.0)$ & $3.0(3.0-3.0)$ & 0.153 \\
Attention & $6.0(5.0-6.0)$ & $6.0(6.0-6.0)$ & 0.030 \\
Language & $2.0(2.0-3.0)$ & $2.0(2.0-3.0)$ & 0.883 \\
Abstraction & $1.0(1.0-2.0)$ & $1.0(1.0-2.0)$ & 0.582 \\
Delayed recall & $2.0(1.0-3.0)$ & $3.0(2.0-3.0)$ & 0.001 \\
Orientation & $6.0(6.0-6.0)$ & $6.0(6.0-6.0)$ & 0.115 \\
\hline
\end{tabular}

Notes: Continuous variables are expressed as mean \pm standard deviation or as median (interquartile range). Categorical variables are expressed as frequency (percent).

Abbreviations: PD, Parkinson's disease; UPDRS III, score of third part of Unified Parkinson Disease Rating Scale; MMSE, Mini-Mental state examination; MoCA, The Beijing version of the Montreal Cognitive Assessment. 
Table 2 Detection threshold and identification threshold of five different types of odors between patients with Parkinson's disease and controls

\begin{tabular}{|c|c|c|c|}
\hline Odor solutions & $\begin{array}{l}\text { PD patients } \\
(n=63)\end{array}$ & $\begin{array}{l}\text { Controls } \\
(n=55)\end{array}$ & $p$-value \\
\hline \multicolumn{4}{|l|}{ Odor detection threshold } \\
\hline$\beta$-phenylethyl alcohol & $5.0(3.0-6.0)$ & $2.0(1.0-3.0)$ & $<0.001$ \\
\hline Methyl cyclopentenolone & $3.0(3.0-5.0)$ & $2.0(1.0-3.0)$ & $<0.001$ \\
\hline Isovaleric acid & $3.0(2.0-5.0)$ & $2.0(2.0-4.0)$ & $<0.001$ \\
\hline$\gamma$-undecalactone & $4.0(3.0-6.0)$ & $3.0(2.0-4.0)$ & 0.001 \\
\hline Skatole (3-methylindole) & $4.0(2.0-6.0)$ & $2.0(1.0-3.0)$ & $<0.001$ \\
\hline \multicolumn{4}{|l|}{ Odor identification threshold } \\
\hline$\beta$-phenylethyl alcohol & $6.0(5.0-6.0)$ & $3.0(2.0-5.5)$ & $<0.001$ \\
\hline Methyl cyclopentenolone & $5.0(4.0-6.0)$ & $2.0(2.0-3.0)$ & $<0.001$ \\
\hline Isovaleric acid & $4.0(3.0-5.5)$ & $3.0(2.0-4.0)$ & $<0.001$ \\
\hline$\gamma$-undecalactone & $5.0(4.0-6.0)$ & $4.0(3.0-4.0)$ & $<0.001$ \\
\hline Skatole (3-methylindole) & $5.0(4.0-6.0)$ & $2.0(1.0-3.0)$ & $<0.001$ \\
\hline
\end{tabular}

Note: Continuous variables are expressed as median (interquartile range).

Abbreviation: PD, Parkinson's disease.

The incidence of hyposmia measured by each kind of odor in patients with PD was statistically higher than in controls (Tables 2 and 3).

ROC analysis revealed that $\beta$-phenylethyl alcohol (with a sensitivity of $87.3 \%$ and specificity of $87.5 \%$ ) and isovaleric acid (with a sensitivity of $83.3 \%$ and specificity of $93.7 \%$ ) were more superior for recognizing odor-identification impairment in early non-demented drug-naïve patients with PD than three other odors. The AUC of $\beta$-phenylethyl alcohol and isovaleric acid were $91.9 \%$ and $90.4 \%$, respectively (Figure 1).

Multivariate logistic regression analysis showed that hyposmia was associated with impaired visuospatial and executive function ( $p=0.013)$, was but independent of other PD variables, such as disease duration and score of UPDRS III after adjusting for age, sex, and cigarette smoking (Table 4).

\section{Discussion}

Our study demonstrated that odors of $\beta$-phenylethyl alcohol (floral smell, smell of rose petals) and isovaleric acid (smell

Table 3 Abnormal identification threshold distribution between patients with Parkinson's disease and controls

\begin{tabular}{|c|c|c|c|}
\hline Odor solutions & $\begin{array}{l}\text { PD patients } \\
(n=63)\end{array}$ & $\begin{array}{l}\text { Controls } \\
(n=55)\end{array}$ & $p$-value \\
\hline$\beta$-phenylethyl alcohol, n (\%) & $56(88.8)$ & $20(36.4)$ & $<0.001$ \\
\hline Methyl cyclopentenolone, n (\%) & $51(81.0)$ & $10(18.2)$ & $<0.001$ \\
\hline Isovaleric acid, n (\%) & $43(68.3)$ & $24(43.6)$ & 0.007 \\
\hline$\gamma$-undecalactone, $n(\%)$ & $52(82.5)$ & $28(50.9)$ & $<0.001$ \\
\hline Skatole (3-methylindole), n (\%) & $49(77.8)$ & $8(14.5)$ & $<0.001$ \\
\hline
\end{tabular}

Abbreviation: PD, Parkinson's disease.

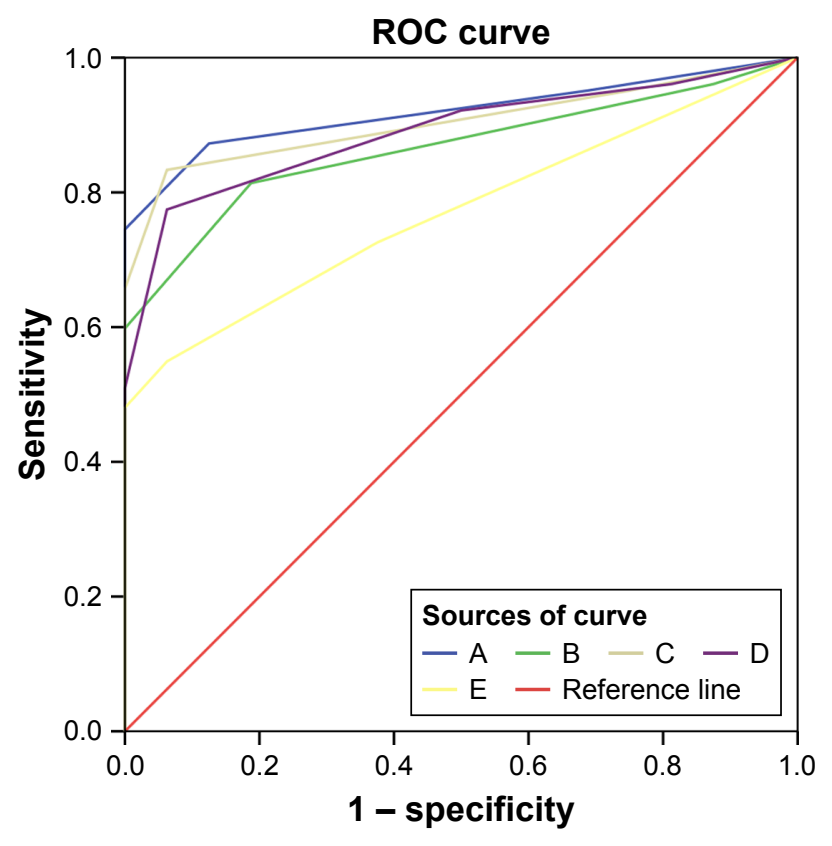

Figure I Receiver operating characteristic (ROC) analysis of each odor for diagnosing hyposmia in patients with Parkinson's disease.

Notes: All $p$-value $<0.001$. Area under the ROC curves of each odor are as follows: A $91.9 \%, 95 \% \mathrm{Cl}(0.869-0.969)$, sensitivity $87.3 \%$, specificity $87.5 \%$; B $86.5 \%, 95 \% \mathrm{Cl}$ (0.796-0.933), sensitivity $81.4 \%$, specificity $81.2 \%$; C $90.4 \%, 95 \% \mathrm{Cl}$ (0.849-0.959), sensitivity $83.3 \%$, specificity $93.7 \%$; D $88.9 \%, 95 \% \mathrm{Cl}(0.826-0.952)$, sensitivity 77.5\%, specificity $93.7 \%$; and $\mathrm{E} 77.1 \%, 95 \% \mathrm{Cl}$ (0.678-0.863), sensitivity 54.9\%, specificity $93.7 \%$.

Abbreviations: $A, \beta$-phenylethyl alcohol; $B$, methyl cyclopentenolone; $\mathrm{C}$, isovaleric acid; D, $\gamma$-undecalactone; E, skatole (3-methylindole).

of sweat, stuffy socks) were more superior for identifying hyposmia in early non-dementia drug-naïve patients with PD. Hyposmia was associated with impaired visuospatial and executive function, but independently of other PDassociated variables, even after adjusting for age, sex, and cigarette smoking.

Table 4 Multivariate logistic regression analysis of predictors for hyposmia in patients with Parkinson's disease

\begin{tabular}{lll}
\hline Variables & $\begin{array}{l}\text { Odds ratio (95\% } \\
\text { confidence interval) }\end{array}$ & -value \\
\hline Sex & $1.899(0.344-10.488)$ & 0.462 \\
Age & $1.052(0.937-1.18 I)$ & 0.393 \\
Disease duration & $0.901(0.642-1.264)$ & 0.546 \\
Cigarette smoking & $0.673(0.131-3.448)$ & 0.634 \\
UPDRS III & $0.966(0.89 I-1.047)$ & 0.403 \\
Visuospatial and executive & $0.527(0.318-0.874)$ & 0.013 \\
Naming & $0.616(0.125-3.04 I)$ & 0.552 \\
Attention & $0.556(0.125-2.467)$ & 0.440 \\
Language & $0.801(0.288-2.229)$ & $0.67 I$ \\
Abstraction & $1.016(0.323-3.195)$ & 0.979 \\
Delayed recall & $0.772(0.475-1.253)$ & 0.295 \\
Orientation & $0.748(0.097-5.744)$ & 0.780 \\
\hline
\end{tabular}

Note: Adjusted for sex, age, disease duration, cigarette smoking, score of UPDRS III, score of visuospatial and executive, naming, attention, language, abstraction, delayed recall, and orientation.

Abbreviation: UPDRS III, score of third part of Unified Parkinson Disease Rating Scale. 
Hyposmia is one of the most commonly seen premotor symptoms in patients with PD. ${ }^{2}$ Mechanisms of hyposmia are currently unknown. PD is a multisystem disorder which involves multiple neurotransmitter pathways within the brain and the autonomic nervous system. ${ }^{24}$ Aside from the dopaminergic system, deficits in the cholinergic, noradrenergic, and serotonergic systems may contribute to olfactory loss. Such deficits, manifesting as decreasing numbers of neurons in the locus coeruleus, raphe nuclei, or nucleus basalis of Meynert, have been correlated with numerous PD-related non-motor symptoms such as autonomic dysfunction as well as hyposmia. ${ }^{5}$ The locus coeruleus, which is also the brain region implicated as being associated with pain and somatosensory disturbances, sends noradrenergic projections to the olfactory bulb and contains a number of olfaction-related forebrain connections. ${ }^{24}$ Activation of 5-hydroxytryptamine fibers originating in the raphe nuclei can alter the output of activity from mitral cells, which are the main projection neurons of the olfactory bulb. ${ }^{25}$ The nucleus basalis of Meynert is the main source of acetylcholine in the forebrain. Evidence suggested that strong correlations between olfactory dysfunction and central cholinergic processes in $\mathrm{PD} .^{26}$ Therefore, hyposmia in PD may have multiple components, including olfactory bulb and primary olfactory cortex components, and indicate limbic dysfunction secondary to dopaminergic, cholinergic, and other denervations.

Hyposmia in patients with PD can present as impairments in odor detection, odor identification, odor discrimination, and odor-recognition memory. The odor-detection threshold is the concentration at which subjects can identify an odor (air, water, and fat). While the odor-identification threshold indicates the level at which a stimulus can not only be detected, but also recognized. Odor-identification testing provides excellent diagnostic accuracy in distinguishing patients with PD from controls. ${ }^{27}$ In this study, the odor-identification threshold was used as the main indicator. Impaired odor identification was the best predictor for future PD. ${ }^{28}$ Ross et $\mathrm{al}^{29}$ carried out a longitudinal Honolulu-Asia Aging Study; 2,267 men, from 1991 to 1996, aged 71 to 95 years who were free of clinical PD and dementia completed the olfaction testing. During a 4-year follow-up period, 35 participants were diagnosed with clinical PD. After adjusting for confounders, compared with those in the two highest quartiles, those with olfactory test scores in the lowest quartile had an OR of 5.2 for developing clinical PD. But, this relation was not found beyond 4 years of follow-up. Hyposmia was correlated with impaired cardiovascular sympathetic function in the early stages of PD and was independent of striatal dopaminergic denervation, disease duration, and clinical ratings of motor function. ${ }^{30}$ Some factors including low protein, folate, magnesium, and phosphorus density of the diet; psychosis; and cognitive function were also shown to be correlated with olfactory dysfunction in patients with PD. ${ }^{31,32}$

The MoCA is one of the most widely adopted screening tools for cognitive impairment and is available in 55 languages. It is a useful screening tool for PD global cognitive and executive functions, and is also highly sensitive for detecting visuospatial and memory impairment in patients with PD. ${ }^{33}$ Mild cognitive impairment (MCI) is a term used to represent a slight cognitive decline which does not fulfil the criteria for dementia. ${ }^{34} \mathrm{MCI}$ in patients with PD covers a wide spectrum of clinical deficits and severity with impairment in language, working memory, attention, executive function, visuospatial abilities, and memory domains - alone or in combination..$^{35}$ Compared to controls, patients with PD exhibited significant deficits in olfactory function and working memory, executive function, speed of information processing, visuospatial skills, and phonological verbal fluency tests. ${ }^{36}$ Loss of awareness of hyposmia was closely associated with MCI in patients with PD. ${ }^{37}$

Recently, several methods of olfactory testing are available in patients with PD including the University of Pennsylvania Smell Identification Test (UPSIT), Barcelona Smell test-24 (BAST-24) for Spain, "Screening 12 Test", and "Sniffin" Sticks'16 (SS-16). The Odor Stick Identification Test for Japanese (OSIT-J) is the only olfactory test designed for the Eastern population among the methods developed in Japan. ${ }^{15}$ Considering that olfactory identification patterns in patients with PD differed among different countries, research on olfactory function in Eastern patients with PD using familiar odors is required..$^{38}$ Few studies have investigated hyposmia in Eastern patients with $\mathrm{PD}$ using familiar odors. Our study showed that hyposmia is common in these early, non-demented, drug-naïve patients with PD. The incidence of hyposmia is significantly higher in patients with PD than in controls. $\beta$-phenylethyl alcohol (floral smell, smell of rose petals) and isovaleric acid (smell of sweat, stuffy socks) were more sensitive for identifying hyposmia in patients with PD than three other odors. The T\&T Olfactometer was also used in another Japanese PD cohort to investigate olfactory dysfunction. Results revealed that methyl cyclopentenolone or skatole had benefits for olfactory evaluation in patients with PD. ${ }^{39}$ Some factors may explain the discrepancy. Firstly, nearly $60 \%$ of their patients were at H\&Y stages III-IV significantly higher than ours. Secondly, there were only 20 controls in their study. Thirdly, the MMSE cut-off value for dementia was defined as 22 , which is lower than the cut-off in this study. In our 
study, patients with PD also had lower scores of visuospatial and executive $(p<0.001)$, attention $(p=0.03)$, and delayed recall ( $p=0.001)$ than controls. Multivariate logistic regression analysis showed that hyposmia was associated with impaired visuospatial and executive function $(p=0.013)$, but independent of other PD-associated variables, such as disease duration and score of UPDRS III after adjusting for age, sex, and cigarette smoking. Visuospatial and executive deficits occur in nearly half of early non-demented patients with PD. ${ }^{40}$ Basal ganglia and limbic structure defects have a determining role in visuospatial impairments in patients with PD. ${ }^{41}$ Basal forebrain cholinergic system degeneration appears to be associated with executive impairment in early PD. ${ }^{42}$ Almost all patients with PD-MCI will eventually fulfil criteria for dementia. ${ }^{34}$ Progressive hyposmia may be a biomarker of cognitive decline and cholinergic denervation in patients with PD. ${ }^{12}$ However, the exact pathological basis between cognitive impairment and hyposmia is unclear. In humans, olfactory information is transmitted from the olfactory bulb to the primary olfactory cortex, including the piriform cortex, amygdala, and entorhinal cortex. In olfactory processes, other cortical areas such as the orbitofrontal and prefrontal areas and the insular, striatum, precuneus, or cerebellum may also be invloved. ${ }^{43}$ Impaired olfactory function is associated with decreased dopaminergic and cholinergic innervation into the temporolimbic areas, and with decreased cerebral metabolism in the piriform cortex and amygdala in PD. ${ }^{10,44}$ Cholinergic denervation of the limbic archicortex is a stronger determinant of hyposmia than nigrostriatal dopaminergic denervation in moderate patients with PD. ${ }^{26}$ The temporolimbic areas together with the orbitofrontal cortex may link olfaction and cognitive function in PD. ${ }^{45}$ Neurodegeneration in dopaminergic, noradrenergic, serotonergic, and especially cholinergic systems in these area appears to play an important role in the correlation between hyposmia and impaired cognitive function in patients with early PD.

In summary, we have demonstrated that olfactory impairment is common in patients with early PD. Compared to three other odors, $\beta$-phenylethyl alcohol and isovaleric acid are relatively more effective in verifying hyposmia in early, non-demented, drug-naïve Chinese patients with PD. Hyposmia was associated with impaired visuospatial and executive function even after adjusting for confounders.

\section{Limitations}

Our study has several limitations. First, as our study is observational in nature, we cannot establish a causal relationship between hyposmia and cognitive dysfunction. Second, the generalizability of our study findings may be limited by the relatively small population from a single center. Moreover, cognitive function of subjects was assessed only by the MoCA rather than a series of neuropsychological tests, such as the Wisconsin Card Sorting Test (CST), Tower of London test-Drexel version, Stroop Test, and Verbal fluency test for executive function testing; Visual Reproduction I and II subtests from the Wechsler Memory Scale, Corsi Learning Suvra-Span, Benton's Judgment of Line Orientation, Hooper Visual Organization Test, and Clock copying for visuospatial function testing.

\section{Acknowledgments}

The authors thank the study participants and their relatives as well as the clinical staff for their support and contribution to this study. The authors also thank David Wu for undertaking language revision. This work was supported by the National Key Plan for R\&D Program of China (2017YFC0909100); Youth fund of Jiangsu Province's Natural Science Foundation (BK20170355); Social development projects in Jiangsu Province (BE2017653); Jiangsu Province's Young Medical Talents Program (QNRC2016872); Suzhou Clinical Research Center of Neurological Disease (Szzx201503); Suzhou Science and Technology Development Program (SYS201620, SYS201624); National Natural Science Pre-Research Foundation of China (SDFEYGJ1605). This study was also partly supported by the Priority Academic Program Development of Jiangsu Higher Education Institutions (PAPD).

\section{Author contributions}

Cheng-Jie Mao and Chun-Feng Liu designed the study. Fen Wang, Ju-Ping Chen, Ya-Ping Yang, Jing Chen, and JuanYing Huang collected the data. Cheng-Jie Mao and Fen Wang analyzed the data and drafted the manuscript, and all authors wrote and revised the article.

\section{Disclosure}

The authors report no conflicts of interest in this work.

\section{References}

1. Driver-Dunckley E, Adler CH, Hentz JG, et al. Olfactory dysfunction in incidental Lewy body disease and Parkinson's disease. Parkinsonism Relat Disord. 2014;20(11):1260-1262.

2. Doty RL, Deems DA, Stellar S. Olfactory dysfunction in parkinsonism: a general deficit unrelated to neurologic signs, disease stage, or disease duration. Neurology. 1988;38(8):1237-1244.

3. Hawkes CH, Shephard BC, Daniel SE. Olfactory dysfunction in Parkinson's disease. J Neurol Neurosurg Psychiatry. 1997;62(5): 436-446. 
4. Doty RL. Olfaction in Parkinson's disease and related disorders. Neurobiol Dis. 2012;46(3):527-552.

5. Doty RL. Olfactory dysfunction in Parkinson disease. Nat Rev Neurol. 2012;8(6):329-339.

6. Roberts RO, Christianson TJ, Kremers WK, et al. Association between olfactory dysfunction and amnestic mild cognitive impairment and Alzheimer disease dementia. JAMA Neurol. 2016;73(1):93-101.

7. Damholdt MF, Borghammer P, Larsen L, Ostergaard K. Odor identification deficits identify Parkinson's disease patients with poor cognitive performance. Mov Disord. 2011;26(11):2045-2050.

8. Yaldizli Ö, Penner IK, Yonekawa T, et al. The association between olfactory bulb volume, cognitive dysfunction, physical disability and depression in multiple sclerosis. Eur J Neurol. 2016;23(3):510-519.

9. Sanke H, Mita T, Yoshii H, et al. Relationship between olfactory dysfunction and cognitive impairment in elderly patients with type 2 diabetes mellitus. Diabetes Res Clin Pract. 2014;106(3):465-473

10. Bohnen NI, Gedela S, Herath P, Constantine GM, Moore RY. Selective hyposmia in Parkinson disease: association with hippocampal dopamine activity. Neurosci Lett. 2008;447(1):12-16.

11. Shimada H, Hirano S, Shinotoh H, et al. Mapping of brain acetylcholinesterase alterations in Lewy body disease by PET. Neurology. 2009; 73(4):273-278.

12. Bohnen NI, Müller ML. In vivo neurochemical imaging of olfactory dysfunction in Parkinson's disease. J Neural Transm (Vienna). 2013; 120(4):571-576.

13. Deems DA, Doty RL, Settle RG, et al. Smell and taste disorders, a study of 750 patients from the University of Pennsylvania Smell and Taste Center. Arch Otolaryngol Head Neck Surg. 1991;117(5):519-528.

14. Wilson RS, Yu L, Bennett DA. Odor identification and mortality in old age. Chem Senses. 2011;36(1):63-67.

15. Rodríguez-Violante M, Gonzalez-Latapi P, Camacho-Ordoñez A, Martínez-Ramírez D, Morales-Briceño H, Cervantes-Arriaga A. Comparing the accuracy of different smell identification tests in Parkinson's disease: relevance of cultural aspects. Clin Neurol Neurosurg. 2014; 123:9-14.

16. Hoehn MM, Yahr MD. Parkinsonism: onset, progression and mortality. Neurology. 1967;17(5):427-442.

17. Hughes AJ, Ben-Shlomo Y, Daniel SE, Lees AJ. What features improve the accuracy of clinical diagnosis in Parkinson's disease: a clinicopathologic study. Neurology. 1992;42(6):1142-1146.

18. Hartmaier SL, Sloane PD, Guess HA, Koch GG, Mitchell CM, Phillips CD. Validation of the Minimum Data Set Cognitive Performance Scale: agreement with the Mini-Mental State Examination J Gerontol A Biol Sci Med Sci. 1995;50(2):M128-M133.

19. Béland SG, Postuma RB, Latreille V, et al. Observational study of the relation between Parkinson's disease and sleep apnea. J Parkinsons Dis. 2015;5(4):805-811.

20. Schab FR. Odor memory: taking stock. Psychol Bull. 1991;109(2): 242-251.

21. Fahn S; Parkinson Study Group. Does levodopa slow or hasten the rate of progression of Parkinson's disease? J Neurol. 2005; 252(Suppl 4):IV37-IV42.

22. Nasreddine ZS, Phillips NA, Bedirian V, et al. The Montreal Cognitive Assessment, MoCA: a brief screening tool for mild cognitive impairment. J Am Geriatr Soc. 2005;53(4):695-699.

23. Kondo H, Matsuda T, Hashiba M, Baba S. A study of the relationship between the T\&T olfactometer and the University of Pennsylvania Smell Identification Test in a Japanese population. Am J Rhinol. 1998;12(5): 353-358.

24. Schapira AHV, Chaudhuri KR, Jenner P. Non-motor features of Parkinson disease. Nat Rev Neurosci. 2017;18(7):435-450.

25. Petzold GC, Hagiwara A, Murthy VN. Serotonergic modulation of odor input to the mammalian olfactory bulb. Nat Neurosci. 2009;12(6): 784-791.
26. Bohnen NI, Müller ML, Kotagal V, et al. Olfactory dysfunction, central cholinergic integrity and cognitive impairment in Parkinson's disease. Brain. 2010;133(Pt 6):1747-1754

27. Mahlknecht P, Pechlaner R, Boesveldt S, et al. Optimizing odor identification testing as quick and accurate diagnostic tool for Parkinson's disease. Mov Disord. 2016;31(9):1408-1413.

28. Ponsen MM, Stoffers D, Twisk JW, Wolters ECH, Berendse HW. Hyposmia and executive dysfunction as predictors of future Parkinson's disease: a prospective study. Mov Disord. 2009;24(7):1060-1065.

29. Ross GW, Petrovitch H, Abbott RD, et al. Association of olfactory dysfunction with risk for future Parkinson's disease. Ann Neurol. 2008; 63(2):167-173.

30. Mizutani Y, Nakamura T, Okada A, et al. Hyposmia and cardiovascular dysautonomia correlatively appear in early-stage Parkinson's disease. Parkinsonism Relat Disord. 2014;20(5):520-524.

31. Ådén E, Carlsson M, Poortvliet E, et al. Dietary intake and olfactory function in patients with newly diagnosed Parkinson's disease: a casecontrol study. Nutr Neurosci. 2011;14(1):25-31.

32. Morley JF, Weintraub D, Mamikonyan E, Moberg PJ, Siderowf AD, Duda JE. Olfactory dysfunction is associated with neuropsychiatric manifestations in Parkinson's disease. Mov Disord. 2011;26(11): 2051-2057.

33. Hendershott TR, Zhu D, Llanes S, Poston KL. Domain-specific accuracy of the Montreal Cognitive Assessment subsections in Parkinson's disease. Parkinsonism Relat Disord. 2017;38:31-34.

34. Janvin CC, Larsen JP, Aarsland D, Hugdahl K. Subtypes of mild cognitive impairment in Parkinson's disease: progression to dementia. Mov Disord. 2006;21(9):1343-1349.

35. Goldman JG, Aggarwal NT, Schroeder CD. Mild cognitive impairment: an update in Parkinson's disease and lessons learned from Alzheimer's disease. Neurodegener Dis Manag. 2015;5(5):425-443.

36. Parrao T, Chana P, Venegas P, Behrens MI, Aylwin ML. Olfactory deficits and cognitive dysfunction in Parkinson's disease. Neurodegener Dis. 2012;10(1-4):179-182.

37. Kawasaki I, Baba T, Takeda A, Mori E. Loss of awareness of hyposmia is associated with mild cognitive impairment in Parkinson's disease. Parkinsonism Relat Disord. 2016;22:74-79.

38. Millar Vernetti P, Rossi M, Cerquetti D, Perez Lloret S, Merello M. Comparison of olfactory identification patterns among Parkinson's disease patients from different countries. Chem Senses. 2016;41(1): 77-83.

39. Kawase Y, Hasegawa K, Kawashima N, Horiuchi E, Ikeda K. Olfactory dysfunction in Parkinson's disease: benefits of quantitative odorant examination. Int J Gen Med. 2010;3:181-185.

40. Pfeiffer HC, Løkkegaard A, Zoetmulder M, Friberg L, Werdelin L. Cognitive impairment in early-stage non-demented Parkinson's disease patients. Acta Neurol Scand. 2014;129(5):307-318.

41. Caproni S, Muti M, Di Renzo A, et al. Subclinical visuospatial impairment in Parkinson's disease: the role of Basal Ganglia and limbic system. Front Neurol. 2014;5:152.

42. Bohnen NI, Albin RL. The cholinergic system and Parkinson disease. Behav Brain Res. 2011;221(2):564-573.

43. Savic I. Imaging of brain activation by odorants in humans. Curr Opin Neurobiol. 2002;12(4):455-461.

44. Baba T, Takeda A, Kikuchi A, et al. Association of olfactory dysfunction and brain. Metabolism in Parkinson's disease. Mov Disord. 2011; 26(4):621-628.

45. Lee JE, Cho KH, Ham JH, Song SK, Sohn YH, Lee PH. Olfactory performance acts as a cognitive reserve in non-demented patients with Parkinson's disease. Parkinsonism Relat Disord. 2014;20(2): 186-191. 


\section{Publish your work in this journal}

Clinical Interventions in Aging is an international, peer-reviewed journal focusing on evidence-based reports on the value or lack thereof of treatments intended to prevent or delay the onset of maladaptive correlates of aging in human beings. This journal is indexed on PubMed Central, MedLine,

CAS, Scopus and the Elsevier Bibliographic databases. The manuscript management system is completely online and includes a very quick and fair peer-review system, which is all easy to use. Visit http://www.dovepress. com/testimonials.php to read real quotes from published authors. 